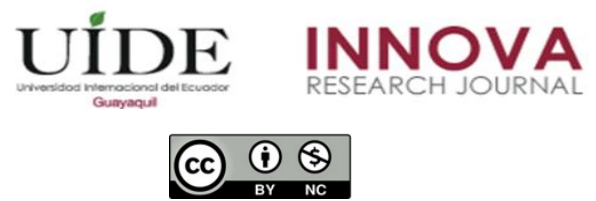

INNOVA Research Journal, ISSN 2477-9024

(Septiembre-Diciembre, 2019). Vol. 4, No.3 pp.170-184

DOI: https://doi.org/10.33890/innova.v4.n3.2019.974

URL: http://revistas.uide.edu.ec/index.php/innova/index

Correo: innova@uide.edu.ec

\title{
Breves consideraciones sobre la Metodología de la Investigación para investigadores principiantes
}

\section{Brief considerations on research Methodology for novice researchers}

\author{
Fabián Darío Rodas Pacheco \\ Juan José Santillán Iñiguez \\ Universidad de Cuenca, Ecuador
}

Autor para correspondencia: fabian.rodas@ucuenca.edu.ec ; juan.santillan@ucuenca.edu.ec Fecha de recepción: 19 de marzo del 2019 - Fecha de aceptación: 11 de julio del 2019

Resumen: Tradicionalmente, la investigación, como actividad científica, ha sido considerada como una tarea exclusiva de grupos académicos de élite; sin embargo, los requerimientos de la sociedad y la Academia actual han originado una mayor necesidad de llevar a cabo estudios científicos y reportarlos en publicaciones académicas. Ante esta realidad, estudiantes y profesionales de todas las áreas del conocimiento, incluyendo, por su puesto a la Educación, se han volcado hacia el desarrollo de procesos de investigación científica. Por esta razón, el presente artículo pretende proveer nociones básicas sobre la Metodología de la Investigación, con el fin de motivar a aquellas personas que están dando sus primeros pasos en este ámbito. Los párrafos subsiguientes presentan una breve introducción al tema, seguida de una reseña histórica, y un análisis sucinto de los distintos métodos de investigación y los componentes de estos procesos, a saber: participantes; recolección, análisis, exploración y representación de datos; al igual que la interpretación y validación de resultados.

Palabras Clave: introducción; metodología de la investigación; investigación cuantitativa; investigación cualitativa; métodos mixtos

\begin{abstract}
Traditionally, research, as a scientific endeavor, has been considered as an exclusive activity for elite academic groups; nevertheless, the current demands of society and Academia have brought about a greater need for developing scientific studies and reporting on them in academic publications. Faced with this reality, students and professionals of all fields of knowledge, including, of course, Education, have turned toward the development of scientific research processes. For this reason, this article offers basic notions regarding Research Methodology, in an attempt to motivate those individuals who are taking their first steps in this field. The paragraphs to follow provide a brief introduction to the topic, and succinct analysis of the diverse research methods and the components of these processes, namely: participants; data gathering, analysis, exploration, and representation; as well as, interpretation and validation of results.
\end{abstract}

Key Words: introduction; research methodology; quantitative research; qualitative research; mixed-methods 


\section{Introducción}

Cuando, en su formación de pregrado, los estudiantes por primera vez se percatan de que existe metodología de la investigación en las Ciencias Sociales, a menudo encuentran términos tales como estadística, confiabilidad y validez, lo que da lugar a que esta área se torne muy compleja, técnica y, por su puesto, intimidante (Dornyei, 2007). También se piensa que investigación la realizan tan solo los expertos y que personas comunes deberían mantenerse al margen de esta actividad. En este escenario, Dornyei (2007) manifiesta para poder producir buenos resultados de investigación, la persona no necesariamente debe tener una vasta experiencia en el área. Para lograr excelencia, a más de rigor académico, se requiere curiosidad genuina, mucho sentido común, buenas ideas, disciplina, confiabilidad y responsabilidad social.

En este contexto, la investigación consiste en encontrar respuestas a preguntas, una actividad que se realiza cotidianamente. Sin embargo, para llevar a cabo una buena investigación, se requiere una adecuada sistematización, lo que permitirá que los resultados sean confiables. Hatch y Lazaraton (1991) sostienen que la investigación, en el sentido científico, consiste en la búsqueda organizada y sistemática de respuestas a las preguntas que realizamos. En esta misma línea, Brown (1988) sugiere dos formas básicas de encontrar respuestas. La primera hace referencia al hecho de averiguar qué es lo que otras personas han investigado sobre ciertos temas; esta forma de indagación recibe el nombre de investigación secundaria, conceptual o bibliográfica, y es una forma esencial de averiguación, ya que sería una pérdida de tiempo y energía ignorar el trabajo de otros autores para intentar reinventar la rueda. La segunda forma de encontrar respuestas consiste en conducir un estudio propio basado en la recolección de datos o información de primera mano, para obtener conclusiones. Este tipo de investigación se conoce con el nombre de investigación empírica.

Con respecto al ámbito de la enseñanza, específicamente en lo relacionado a idiomas extranjeros, a pesar de la gran cantidad de estudios realizados y publicados, el docenteinvestigador todavía puede contribuir a su área, ya que las situaciones de aprendizaje no siempre son las mismas; por lo tanto, la generalización no es una característica de la investigación en las Ciencias Sociales. Además, existen avances en investigación que se construyen en base a resultados de estudios pasados (Dornyei, 2007).Es importante señalar, asimismo, que la investigación no sólo consiste en buscar respuestas a ciertas preguntas, sino también se convierte en una de las formas más efectivas de desarrollo profesional que beneficia tanto al investigador como a otras personas. McKay (2006) sostiene que, para los docentes, una de las principales razones para realizar investigación es el convertirse en mejores maestros. En este ámbito, la investigación contribuye a una enseñanza más efectiva, no solo ofreciendo respuestas definitivas a preguntas pedagógicas, sino dando nuevas orientaciones al proceso de enseñanza aprendizaje.

Como respuesta a esta discusión, lo que se pretende alcanzar con el presente artículo es la provisión de una discusión concisa de las nociones y conceptos básicos de la Metodología de la Investigación, a fin de facilitar su comprensión, especialmente en investigadores nóveles. Por este motivo, los párrafos que se ponen a consideración dan a conocer una sucinta reseña histórica de la Metodología de la Investigación en general, para luego continuar con el análisis de aspectos específicos relacionados con los métodos y el diseño de un estudio. 


\section{Breve Reseña Histórica}

La investigación social cuantitativa originalmente nace como respuesta a los resultados empíricos de las Ciencias Naturales en el siglo XIX. Una de las consecuencias de este hecho fue que los primeros investigadores sociales se propusieran adoptar en sus estudios lo que en aquella época se conocía como el Método Científico.

Este método ha venido evolucionando en el pensamiento occidental desde la mitad del siglo XVI, es decir, desde el Período de la Ilustración, a través del trabajo de filósofos y estudiosos como Copérnico, Bacon, Galileo, Kepler, Newton, Descartes, Hume, Compte, y Pierce (Garrat \& Li, 2005). Básicamente, el Método Científico postula tres etapas elementales en el proceso de investigación: observación del fenómeno o identificación de un problema, la creación de una hipótesis inicial, y la comprobación de la hipótesis por medio de la recolección y análisis de datos empíricos, utilizando procedimientos estandarizados. Cuando la hipótesis ha sido exitosamente comprobada y posteriormente validada a través de un proceso de replicación, ésta es aceptada como una teoría o ley científica. Al mismo tiempo, el método científico ofrece una herramienta para explorar preguntas de una manera objetiva, minimizando la influencia del sesgo o prejuicio del investigador; el resultado, por lo tanto, según los estudiosos del área, es una descripción precisa y confiable del mundo que nos rodea (Dornyei, 2007).

El Método Científico estaba bastante asociado con valores numéricos y con la estadística. En este contexto, Lord Rutherford solía decir que cualquier conocimiento que no podía ser medido numéricamente era un conocimiento pobre; por lo tanto, para que una persona pueda ser un científico, era necesario que tenga la capacidad de medir empíricamente el objeto de estudio $y$, preferiblemente, realizar experimentos. En este escenario, para poder cumplir con las exigencias cuantitativas que la popularidad del Método Científico impuso a las emergentes Ciencias Sociales, la Estadística se convirtió en una subdisciplina integral de las Matemáticas a finales del siglo XIX. En este contexto, la contribución del académico Francis Galton fue significativa, ya que estableció métodos de recolección y análisis de datos cuantitativos en la Psicología a finales del siglo XIX. De esta manera, Galton introdujo las pruebas psicológicas, el uso de cuestionarios, y creó los conceptos estadísticos de regresión y correlación.

A mediados del siglo XX, se dieron una gran cantidad de avances tanto en el Método Científico, básicamente a través del trabajo de Karl Popper, como en la Estadística, con Spearman, Fisher, Nyeman y Pearson; es hecho dio lugar al uso creciente de la metodología cuantitativa en las Ciencias Sociales (Dornyei, 2007). Como resultado de este progreso, las Ciencias Sociales adquirieron madurez y obtuvieron la reputación de ser capaces de estudiar al ser humano científicamente tanto a nivel individual como a nivel de sociedad. A mediados del siglo XX, y debido a los avances en la Psicometría - una subdisciplina de la Psicología que se centra en la medición - la teoría clásica de las pruebas, el diseño experimental, la investigación por encuestas, la teoría sobre cuestionarios y la estadística multivariable, es decir, la metodología cuantitativa se tornó dominante en las Ciencias Sociales. Sin embargo, esta hegemonía empezó a cambiar la década de los setenta como resultado de los retos y avances de la investigación cualitativa, dando lugar a una reestructuración en la metodología de la investigación. Actualmente, en varias áreas de las Ciencias Sociales, se puede notar una coexistencia pacífica entre el método cuantitativo y el cualitativo. 
Con respecto a la Lingüística Aplicada, Lazaraton (2005) manifiesta que durante el período de 1970 y 1985 se dio un significativo incremento en la publicación de artículos con enfoque cualitativo; este incremento tuvo lugar a la par con publicaciones de varios textos sobre métodos de investigación en los años ochenta. No obstante, el enfoque cuantitativo todavía era dominante. De hecho, Hatch y Lazaraton (1991), en su obra pionera, Manual de Investigación, proporcionan un resumen detallado sobre investigación cuantitativa y estadística, mismo que estuvo ricamente ilustrado con estudios previamente publicados. Lazaraton (2005) adicionalmente informa sobre una investigación que analizó 524 estudios empíricos que aparecieron en cuatro grandes revistas de Lingüística Aplicada entre 1991 y 2001. Los resultados de este estudio revelaron que el $86 \%$ de los artículos de investigación fueron cuantitativos, $13 \%$ cualitativos y tan sólo el $1 \%$ de metodología mixta.

Empero, Lazaraton (2005) también resalta el gran cambio que se ha dado con respecto a investigación en Lingüística Aplicada; mientras que, en los años ochenta, existió una dependencia incuestionable en estudios cuasi experimental, en los últimos años, se ha podido notar una perspectiva multidisciplinaria con respecto a metodología de la investigación, con un creciente número de alternativas especialmente cualitativas.

Varios estudiosos de las Ciencias Sociales han venido utilizando datos cuantitativos al igual que datos cualitativos desde inicios del siglo XX. No obstante, la distinción cuantitativacualitativa empieza luego de que la investigación estadística basada en números se vuelve dominante a mediados del siglo XX, dando lugar a que ciertos académicos empiecen a cuestionar esta hegemonía, defendiendo de esta manera el enfoque cualitativo. En este escenario, los términos "cuantitativo" y "cualitativo" emergen como resultado de esta confrontación.

Por su parte, Schwandt (2000) describe el enfoque cualitativo como un movimiento reformista que reúne a una gran variedad de estudiosos que comparten muy poco en común, excepto por su desagrado por el paradigma cuantitativo. En esta misma línea, Dornyei (2007) menciona que un aspecto que es común a los dos enfoques es la gran cantidad de información que se obtiene del mundo social que nos rodea, misma que debe reducirse con el propósito de que sea manejable.

\section{Tipos de metodología}

Una de las distinciones más generales y más conocidas en metodología de la investigación es la que existe entre la metodología cuantitativa y cualitativa. Dornyei (2007) presenta definiciones preliminares sobre estos dos enfoques. Por un lado, señala que la investigación cuantitativa involucra procedimientos de recolección de datos que dan lugar principalmente a información numérica que luego es analizada por medios estadísticos. Por otro lado, Dornyei (2007) sostiene que la investigación cualitativa requiere procedimientos de recolección de datos que resultan principalmente en información abierta y no numérica la que es analizada a través de métodos no estadísticos.

Bernal (2013) añade que la investigación cualitativa busca prioritariamente la descripción más no la generalización. Sin embargo, Bloor (2016) afirma que, además de la posibilidad de influenciar la creación y reforma de políticas, la investigación cualitativa, especialmente dentro 
de las Ciencias Sociales, permite identificar y tratar problemas sociales, al incluir participativamente dentro del proceso de investigación a todos los actores involucrados en el fenómeno de estudio.

Por su parte, Davies (1995) indica que la diferencia representa algo más que tan solo el uso de figuras numéricas versus datos no cuantitativos tales como entrevistas abiertas o datos naturales. La dicotomía hace referencia a varios aspectos al mismo tiempo, por ejemplo, la orientación ideológica general detrás del estudio, el método de recolección de datos utilizado, la naturaleza de la información recolectada y el método de análisis usado para procesar los datos y obtener resultados.

Dentro de esta discusión, Sánchez Gómez (2015) comenta sobre la postura de la armonía, $\mathrm{y}$, desde esta perspectiva, indica que:

Se [deben valorar] igualmente las posibilidades de cada metodología, respetando sus respectivas contribuciones. Esto no implica que se conceda a la una y a la otra más valía global, sino que [la elección] suele ceñirse a espacios sociales específicos que le son propios y, específicamente ajustados a sus características genuinas. La idea es, por tanto, adoptar una postura dual, esto es, asumir la dualidad sin sacrificar ninguno de los dos extremos. (p.16)

En este ámbito, y para que exista un balance metodológico, entra en juego el Método Mixto que consiste en una serie de combinaciones de investigación cuantitativa y cualitativa, ya sea a nivel de recolección de datos o de análisis de estos. Johnson y Christensen (2004) mencionan que una metodología de investigación mixta está conformada por la mezcla de métodos o paradigmas tanto cuantitativos como cualitativos. Por su parte, Gheitasi y Lindgren (2015) conectan a los estudios de métodos mixtos con un paradigma de la investigación científica en el que las decisiones son respecto al diseño del estudio no son hechas en obediencia a compromisos filosóficos, sino en base a su idoneidad para los propósitos y objetivos del estudio. Los mismos autores señalan que el principio subyacente de la metodología mixta es que, al combinar los métodos cuantitativo y cualitativo, los investigadores pueden tomar de las fortalezas de cada uno para compensar así por sus debilidades. Esta metodología hoy en día es de especial importancia para el fortalecimiento de la investigación educativa, tratando de evitar que un estudio de esta naturaleza se polarice en la elección de un solo paradigma, ya sea cuantitativo o cualitativo.

Adicionalmente, Mertens (2005) da a conocer que la metodología mixta tiene un valor particular cuando se trata de examinar un tema que está inmerso en un contexto social o educativo complejo. En esta misma línea, Onwuegbuzie y Leech (2005) añaden que actualmente los estudios que utilizan un solo método de investigación se convierten en una amenaza para el adelanto de las Ciencias Sociales. En esta misma línea de pensamiento, Brown (2014) afirma que, dado que la investigación de Métodos Mixtos adquirió aceptación y popularidad de manera reciente, y dado que, a pesar de que combina características y estándares cuantitativos y cualitativos, la investigación de Métodos Mixtos es más que la suma de estas partes discretas, es necesario que los investigadores de este tipo de metodología piensen y describan en sus estudios los aspectos de la investigación de Métodos Mixtos que son únicos, y por eso, valiosos. 
Según Sandelowsky (2003), existen dos propósitos principales para combinar métodos: el primero tiene que ver con el logro de una mejor comprensión del fenómeno en estudio y el segundo con la verificación de un grupo de resultados con respecto del otro. Sobre estos dos propósitos, Dornyei (2007) manifiesta que el primero pretende lograr una comprensión significativa de un asunto complejo, mirándolo desde diferentes ángulos; el segundo propósito está relacionado con el objetivo tradicional de la triangulación de datos para validar conclusiones a través de la presentación de resultados convergentes obtenidos por medio de diferentes métodos.

Flick (2014) sostiene que la triangulación involucra la combinación de varias estrategias y métodos cuantitativos y cualitativos, permitiendo de estas maneras que las debilidades de cada método, tomados de manera aislada, se compensen. Asimismo, Dornyei (2007) mantiene que la triangulación de datos ha jugado un rol importante en las Ciencias Sociales, introduciendo el uso combinado de investigación cuantitativa y cualitativa en los años 70. Desde entonces, la triangulación ha sido vista como una estrategia efectiva para asegurar la validez de la investigación. Si los resultados sobreviven una serie de pruebas con diferentes métodos, éstos pueden ser considerados mucho más válidos que una hipótesis que ha sido verificada a través de un solo método. (Erzberger \& Kelle, 2003).

\section{Participantes}

Dornyei (2007) sostiene que la muestra es el grupo de participantes a quienes el investigador en realidad examina en un estudio empírico, mientras que la población está conformada por el grupo de personas a quienes se refiere la investigación. En este acápite, los investigadores detallan las características más importantes de los participantes: el número de personas de la muestra, la edad, el género, la clase social, el nivel de educación, la etnia a la que pertenecen, el idioma materno, nivel de dominio del segundo idioma, etc. Es necesario recalcar que, como Mackey y Gass (2005) mencionan, las características que los investigadores describan en sus reportes dependerán del tipo de estudio que se está llevando a cabo.

Con respecto al número de participantes, éste depende del tipo de metodología sobre la que está basada la investigación. Creswell y Plano Clark (2011) sostienen que, en investigación cualitativa, en lugar de seleccionar una muestra amplia, el investigador cualitativo recluta un número pequeño, mismo que brindará información a fondo sobre el fenómeno central o concepto que está siendo explorado en el estudio. Estos autores además indican que la idea en investigación cualitativa no es generalizar a partir de la muestra, como lo es en investigación cuantitativa, sino desarrollar una comprensión a fondo de unas pocas personas, ya que mientras más grande sea el número, menos información emergerá de cada una de ellas. De hecho, varios investigadores cualitativos prefieren no reportar números exactos con respecto a las muestras de sus estudios; por su parte, Creswell (2007) sugiere que, dependiendo de la naturaleza de la investigación, un número pequeño es aceptable, entre 4 y 10 participantes. Finalmente, este autor sostiene que el tamaño de la muestra dependerá de los tipos de enfoques cualitativos utilizados, mismos que pueden ser narrativos, fenomenológicos, etnográficos, teoría fundamentada o estudios de caso. 
Se debe mencionar, además, que, dentro de la investigación en Ciencias Sociales, un procedimiento de selección de muestra que ha ganado popularidad es el muestreo por conveniencia. Etikan, Abubakar, Sunusi (2016) se refieren al muestreo por conveniencia como uno en el que los participantes del estudio son seleccionados por cumplir ciertos criterios prácticos, como facilidad de acceso, proximidad geográfica, disponibilidad, y voluntad misma de participación. Es importante mencionar que, a pesar de que el muestreo por conveniencia puede ser visto como un factor que imposibilita la generalización de resultados, su importancia en las Ciencias Sociales radica en el hecho de que la investigación en éstas no tiene a la generalización como uno de sus objetivos fundamentales (Bernal, 2013).

\section{Instrumentos a utilizarse}

Brown (2014) afirma que tomar decisiones correctas con respecto al tipo de instrumentos que se van a utilizar en una investigación puede requerir de mucho tiempo y consideración. Por otro lado, a más de decidir qué instrumentos darán a conocer información confiable, es necesario buscar la forma de reunir datos que finalmente sean fáciles y eficientes al momento de recopilarlos y analizarlos.

Uno de los instrumentos más utilizados en investigación es el cuestionario, mismo que forma parte de la encuesta, cuyos resultados son típicamente cuantitativos, con excepción de aquellos que contengan preguntas abiertas, lo que requeriría de un análisis cualitativo. Dornyei (2007) indica que la popularidad de los cuestionarios se debe al hecho de que éstos son fáciles de elaborar, extremadamente versátiles y capaces de reunir una gran cantidad de información de manera rápida y accesible para su procesamiento.

Con respecto a las entrevistas, Miller y Glassner (2016) señalan que, si bien es cierto que no se puede afirmar que las entrevistas sean un método de recolección de datos en contextos naturales, estas, cuando son planificadas y aplicadas pertinentemente, tienen la capacidad de proveer descripciones auténticas de los fenómenos sociales que se estudian. Por su parte, Mackey y Gass (2005) dan a conocer que existen varias clases que pueden ser utilizadas para obtener información como parte del método cualitativo. Estas pueden ser entrevistas estructuradas, semiestructuradas y no estructuradas.

Las entrevistas estructuradas son aquellas en las que el investigador usualmente realiza las mismas preguntas a todos los participantes. Estas preguntas son similares a cuestionarios orales. En cuanto a las entrevistas semiestructuradas, el investigador utiliza una lista escrita de preguntas como guía, pero tiene la libertad de referirse a otros temas. Es importante mencionar que este tipo de entrevista es una de las más utilizadas en Lingüística Aplicada y en el área educativa en general. Además, se constituye un punto medio entre los dos extremos. En este escenario, el entrevistador proporciona una guía e indica el camino, pero también se muestra predispuesto a seguir el desarrollo de la entrevista, permitiendo que el entrevistado se exprese sobre ciertos temas de su interés (Mackey \& Gass, 2005).

Finalmente, las entrevistas no estructuradas permiten al investigador trabajar en base a ciertas preguntas que den lugar a que los participantes se abran al diálogo y se expresen en sus propios términos y ritmos. En este contexto, Miller y Crabtree (1999) manifiestan que la 
entrevista con sus diferentes convenciones, expectativas, etiqueta e inclusive las frases lingüísticas es un conocimiento cultural compartido de los participantes.

Seidman (1998) recomienda a los investigadores que administren una secuencia de tres entrevistas con los mismos participantes para obtener suficiente información. La primera entrevista se aplica con el propósito de romper el hielo y crear un ambiente de empatía, a la vez que se realiza un rápido barrido de las áreas que serán investigadas posteriormente. El intervalo entre la primera y la segunda entrevista permite al investigador preparar una mejor guía para la segunda sesión y brinda la oportunidad al entrevistado para que se involucre de una mejor manera en esta nueva experiencia. Como resultado de esta preparación previa, la segunda entrevista se lleva a cabo sin mayores novedades, concentrándose en los temas de interés de la investigación. Luego del respectivo análisis de las transcripciones de las dos primeras entrevistas, el propósito de la tercera, según Seidman (1998), es cubrir ciertos temas que no fueron tratados en las sesiones previas. Finalmente, el autor recalca que tanto los cuestionarios como las entrevistas semiestructuradas deben ser piloteados con cierta anticipación, previo a su aplicación definitiva.

\section{Recolección de Datos}

El objetivo con respecto a recolección de datos en cualquier investigación consiste en reunir información para abordar las preguntas del estudio que se está realizando. Por ejemplo, en la metodología mixta, el procedimiento de recolección de datos está formado por ciertos componentes clave como el muestreo, la obtención de permisos, la recolección de datos en sí, la grabación de los datos y la administración de procedimientos de recolección de datos que involucra acciones específicas que toma el investigador para el acopio de la información (Creswell \& Plano Clark, 2011). En este contexto, la recolección de datos significa un proceso con varios pasos interconectados entre sí. En esta misma línea, Creswell y Plano Clark (2011) mencionan que, en la metodología mixta, la recolección de datos requiere que se proceda a través de dos corrientes: la cuantitativa y la cualitativa. Cada una de ellas necesita ser totalmente ejecutada por medio de enfoques minuciosos para asegurar la rigurosidad de la investigación.

Creswell y Plano Clark (2011), luego de recopilar información sobre varios estudios con metodología mixta, recomiendan trabajar con ciertos componentes, mismos que se dan detallan a continuación:

Tabla 1. Recopilación de datos en metodología mixta (Creswell \& Plano Clark, 2011).

\begin{tabular}{|c|c|c|}
\hline $\begin{array}{l}\text { Procedimientos de Recolección } \\
\text { de Datos Cualitativos } \\
\text { Convincentes }\end{array}$ & $\begin{array}{l}\text { Procedimientos en la } \\
\text { Recolección de Datos }\end{array}$ & $\begin{array}{c}\text { Recolección de Datos } \\
\text { Cuantitativos Rigurosos }\end{array}$ \\
\hline $\begin{array}{ll}\text { - } & \text { Identifique el lugar o los } \\
\text { lugares que van a ser } \\
\text { estudiados. } \\
\text { - Identifique los } \\
\text { participantes del } \\
\text { estudio. }\end{array}$ & $\begin{array}{l}\text { Uso de procedimientos de } \\
\text { muestreo }\end{array}$ & $\begin{array}{l}\text { - Identifique el lugar o los } \\
\text { lugares que van a ser } \\
\text { estudiados. } \\
\text { - Identifique los } \\
\text { participantes del } \\
\text { estudio. }\end{array}$ \\
\hline
\end{tabular}




\begin{tabular}{|c|c|c|}
\hline $\begin{array}{l}\text { Procedimientos de Recolección } \\
\text { de Datos Cualitativos } \\
\text { Convincentes }\end{array}$ & $\begin{array}{l}\text { Procedimientos en la } \\
\text { Recolección de Datos }\end{array}$ & $\begin{array}{c}\text { Recolección de Datos } \\
\text { Cuantitativos Rigurosos }\end{array}$ \\
\hline $\begin{array}{l}\text { - Anote el tamaño de la } \\
\text { muestra. } \\
\text { - Identifique la estrategia } \\
\text { de muestreo intencional } \\
\text { para enrolar a los } \\
\text { participantes e indique } \\
\text { por qué fue escogida. } \\
\text { Discuta las estrategias } \\
\text { de selección de los } \\
\text { participantes. }\end{array}$ & & 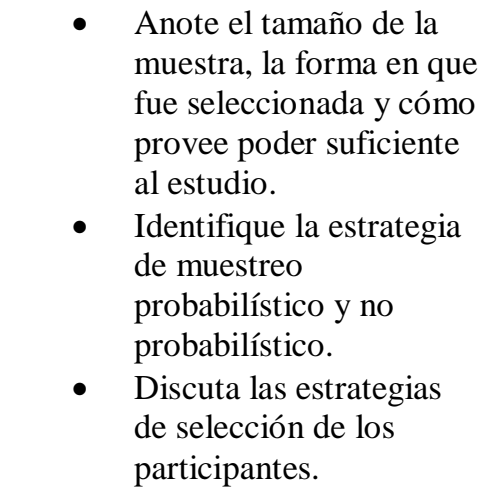 \\
\hline $\begin{array}{l}\text { - Analice los permisos } \\
\text { requeridos para } \\
\text { investigar los lugares y } \\
\text { los participantes. } \\
\text { Obtenga permisos del } \\
\text { Consejo de Revisión } \\
\text { Institucional. } \\
\end{array}$ & Obtención de Permisos & $\begin{array}{l}\text { Analice los permisos } \\
\text { requeridos para } \\
\text { investigar los lugares y } \\
\text { los participantes. } \\
\text { Obtenga permisos del } \\
\text { Consejo de Revisión } \\
\text { Institucional. } \\
\end{array}$ \\
\hline 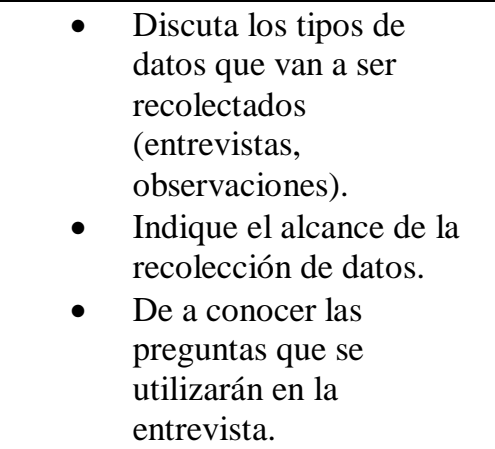 & Recolección de la Información & $\begin{array}{l}\text { Discuta los tipos de } \\
\text { datos que van a ser } \\
\text { recolectados } \\
\text { (instrumentos, } \\
\text { observaciones, registros } \\
\text { cuantificables). } \\
\text { Indique los resultados } \\
\text { reportados sobre validez } \\
\text { y confiabilidad sobre los } \\
\text { instrumentos utilizados. }\end{array}$ \\
\hline $\begin{array}{l}\text { - } \quad \text { Mencione qué } \\
\text { protocolos serán } \\
\text { utilizados (entrevistas, } \\
\text { observaciones). } \\
\text { Identifique los métodos } \\
\text { de registro de los datos. }\end{array}$ & Registro de los Datos & $\begin{array}{l}\text { - Mencione qué } \\
\text { instrumentos o listas de } \\
\text { cotejos serán utilizados } \\
\text { y de ejemplos. }\end{array}$ \\
\hline $\begin{array}{l}\text { - Identifique cuestiones de } \\
\text { recopilación de datos } \\
\text { previos } \\
\text { (Éticas, logísticas). }\end{array}$ & $\begin{array}{l}\text { Aplicación de los } \\
\text { Procedimientos }\end{array}$ & $\begin{array}{ll}\text { - } & \text { Indique cómo los } \\
\text { procedimientos serán } \\
\text { estandarizados. } \\
\text { - Identifique cuestiones } \\
\text { éticas previas. }\end{array}$ \\
\hline
\end{tabular}

\section{Análisis de los datos}

Luego de que se han recolectado los datos, el siguiente paso consiste en analizarlos. Para ello, dependiendo del tipo de información con la que se vaya a trabajar, se requiere del uso de la estadística y de cierto software especializado. Mackey y Gass (2005) recomiendan a los investigadores novatos o principiantes que antes de conducir un análisis estadístico de datos, se 
familiaricen con la estadística de su estudio a través de cursos, textos o consultorías con expertos en el área.

Por otro lado, es importante mencionar que, hace varios años atrás, los datos estadísticos eran calculados manualmente en papel, llenando varias hojas con ecuaciones matemáticas y con la ayuda de una simple calculadora. No obstante, actualmente, esta situación ha cambiado completamente, pues los aspectos matemáticos de análisis de datos cuantitativos los manejan las computadoras y varios programas estadísticos especializados han sido desarrollados para guiar el trabajo de manera computarizada.

En cuanto al campo informático, existen muchos programas estadísticos en el mercado, algunos de ellos son muy amigables y no tan complicados de usar. En este ámbito, Dornyei (2007) sugiere a los investigadores que no tienen mucha experiencia seleccionar ciertos programas con el propósito de familiarizarse con ellos y empezar a utilizarlos. Creswell y Plano Clark (2011) mencionan que uno de los paquetes de software estadísticos más comúnmente utilizados en Linguística Aplicada y en investigación educativa es el Paquete Estadístico para las Ciencias Sociales (SPSS, por sus siglas en inglés). Con respecto a este software, Dornyei (2007) acota que, si bien el SPSS no es para principiantes, aquellos investigadores que tienen cierta experiencia en estadística y programación no tendrían mayores dificultades al implementarlo ya que presenta varias características interactivas y amigables.

Por otro lado, Brown (2001) da conocer que la recolección de datos es tan solo la mitad de la batalla, pues se requiere el procesamiento de los mismos. Para ello, es necesario convertir las respuestas de los participantes en números a través de ciertos procedimientos de codificación. Debido a que los números en sí no tienen significado y son fáciles de mezclarlos y confundirlos, un elemento importante de esta etapa de codificación consiste en definir cada variable para luego compilar valores que cada una podría tener (Dornyei, 2007). Por ejemplo, cuando se trabaja con información sobre género, ésta usualmente va etiquetada como "sexo" y puede tener dos valores numéricos, masculinos con el código " 1 " y femeninos con el "2". Es importante mencionar que aquellos cuestionarios que contienen preguntas cerradas son más sencillos de codificar que aquellos que contienen preguntas abiertas, para cuyas respuestas es necesario un cierto grado de interpretación por parte del investigador.

Adicionalmente, este proceso consiste en examinar la base de datos para orientar las preguntas de investigación o las hipótesis. En un análisis de datos cuantitativos, el investigador revisa los datos basados en el tipo de preguntas o hipótesis y utiliza el test estadístico apropiado para guiarlos. Según Creswell y Plano Clark (2011), la elección del test estadístico está basada en el tipo de preguntas realizadas. Estos autores también sugieren a los investigadores buscar evidencia sobre resultados prácticos, reportados como medidas de efecto e intervalos de confidencia. El análisis de datos cuantitativos procede de un análisis inferencial, dando lugar a que varios pasos de este proceso permitan construir un análisis más refinado.

Con respecto al análisis de datos cualitativos, éste involucra dividir un texto información recolectada - en unidades más pequeñas tales como frases, oraciones o párrafos. Luego se pone una etiqueta en cada unidad, para finalmente agrupar los códigos en temas. Esta codificación se la puede hacer manualmente a través de la asignación de códigos que van 
ubicados tanto en el margen izquierdo como en el derecho de las páginas transcritas. Empero, hoy en día se puede hacer uso de varios programas informáticos que pueden almacenar documentos para su respectivo análisis, tal como Atlas.ti y NVivo. Estos programas también permiten que el investigador pueda bloquear y etiquetar partes de textos con códigos, mismos que pueden ser fácilmente recuperados. Adicionalmente, dichos programas pueden organizar los códigos, permitiendo diagramar y observar la relación que existe entre ellos al igual que buscar partes del texto que contienen códigos múltiples (Creswell \& Plano Clark, 2011).

\section{Exploración de datos}

Creswell y Plano Clark (2011) recomiendan también explorar los datos. En análisis cuantitativo de datos, esto significa inspeccionar los datos visualmente y conducir un análisis descriptivo, mismo que tiene que ver con la media, la desviación estándar y la varianza de respuestas de cada ítem, con el propósito de determinar las orientaciones generales de los datos. Los investigadores exploran los datos para ver su distribución y determinar si son normalmente o anormalmente distribuidos para poder seleccionar el medio estadístico apropiado para su análisis.

Por otro lado, la exploración de datos en el análisis cualitativo involucra una lectura completa de toda la información con el propósito de desarrollar un entendimiento general de la base de datos. Esto significa registrar ideas iniciales a través de la escritura de memorandos cortos en los márgenes de las transcripciones o notas de campo (Creswell \& Plano Clark, 2011). Asimismo, es importante anotar que todo tipo de información es revisada, por ejemplo, notas de observación de campo, diarios, actas de reuniones, fotografías y transcripciones de entrevistas. La elaboración de memorandos se convierte en un paso fundamental en la formación de categorías más amplias de información tales como los códigos o temas. Finalmente, en esta etapa, es necesario crear un libro de códigos cuyo objetivo es ayudar a organizar la información a medida que nuevos códigos son agregados y otros removidos.

\section{Representación del análisis de los datos}

A continuación, el siguiente paso en el proceso de análisis consiste en representar los resultados del análisis en forma resumida por medio de oraciones, tablas o figuras. Los resúmenes pueden ser oraciones que sinteticen los resultados. Las tablas en investigación cuantitativa pueden reportar resultados relacionados con preguntas descriptivas o preguntas inferenciales. Estas tablas requieren estar bien organizadas, detallando con claridad el título, las filas y columnas etiquetadas. Con respecto a las figuras, los investigadores las utilizan para representar resultados cuantitativos de manera visual, mismos que pueden ser gráficos de barras, diagramas o gráficos de dispersión, gráficos lineales y cuadros.

En cuanto a la investigación cualitativa, la representación de datos puede involucrar una discusión de la evidencia para los temas o categorías, la representación de figuras que dan a conocer el lugar físico del estudio o diagramas que presentan marcos de referencia, modelos o teorías. La idea básica cuando se discute la evidencia de un tema o categoría es elaborar una discusión que convenza al lector que el tema o la categoría proviene de los datos (Creswell \& Plano Clark, 2011). Asimismo, los investigadores pueden dar a conocer los hallazgos por medio 
de figuras, mapas o tablas que representan a los diferentes temas tratados. Adicionalmente, se puede incluir extractos de respuestas a entrevistas con el fin de ilustrar las ideas que se discuten.

\section{Interpretación de Resultados}

Luego de representar los resultados o hallazgos, el investigador procede a realizar una interpretación del significado de los resultados, lo que usualmente aparece en la sección llamada discusión de un informe. Creswell y Plano Clark (2011) manifiestan que la interpretación de los resultados significa regresar a los resultados detallados y avanzar con mayor significado, en base a los problemas de investigación, las preguntas de un estudio y la literatura existente. Con respecto a investigación cuantitativa, esto significa comparar los resultados con las preguntas de investigación iniciales con el propósito de determinar cómo las preguntas o hipótesis fueron respondidas en el estudio. También significa comparar los resultados con predicciones anteriores, las que dan explicaciones a lo que el investigador ha encontrado.

Por otro lado, en investigación cualitativa la interpretación arroja similares explicaciones sobre los resultados, excepto por unas pocas diferencias. Creswell y Plano Clark (2011) sostienen que en este tipo de estudio, el investigador requiere reportar cómo la preguntas de investigación fueron contestadas por medio de los hallazgos cualitativos. Adicionalmente, estos autores mencionan que los investigadores cualitativos pueden incluir sus experiencias personales y hacer evaluaciones de los significados de los hallazgos.

\section{Validación de datos y resultados}

Otro componente que forma parte de una buena investigación consiste en utilizar procedimientos para asegurar la validez de los datos, de los resultados y de su interpretación (Creswell \& Plano Clark, 2011).

Creswell (2009) sostiene que, en investigación cuantitativa, el investigador se preocupa de dos temas de validez: la calidad de los resultados de los instrumentos usados y la calidad de las conclusiones obtenidas de los resultados del análisis cuantitativo. En este contexto, validez cuantitativa quiere decir que las respuestas de los participantes son indicadores significativos del constructo que se está midiendo.

Por otro lado, Creswell (2009) sostiene que, para evaluar la validez de un estudio, los investigadores establecen la validez de los instrumentos a través de validación de contenido; y de las respuestas, por medio de procedimientos de criterios relacionados y de la validez del constructo. Asimismo, según este autor, los investigadores cuantitativos también consideran la validez de las conclusiones que obtienen de los resultados. Finalmente, los investigadores cuantitativos toman en cuenta aspectos relacionados con la confiabilidad, lo que significa que las respuestas recibidas por los participantes son consistentes y estables a lo largo del tiempo.

En lo que a investigación cualitativa se refiere, el énfasis se centra más en la validez que en la confiabilidad, con objeto de determinar si el reporte presentado por los participantes y por el investigador es preciso, confiable y fidedigno (Lincoln \& Guba, 1985). En este ámbito, la validez cualitativa proviene de procedimientos de análisis del investigador, los que se basan en 
información recogida mientras se conversa con los participantes, además de aquella proveniente de evaluadores externos. En cuanto a confiabilidad, Lincoln y Guba (1985) manifiestan que ésta juega un rol menor en investigación cualitativa, debido a que la confiabilidad está relacionada con codificadores múltiples de un equipo con el propósito de alcanzar acuerdos sobre códigos de pasajes de textos.

En este ámbito, es importante establecer la validez cualitativa, lo que significa valorar si la información obtenida a través de datos cualitativos es precisa. Creswell (2007) manifiesta que hay varias estrategias para determinar la validez de un estudio cualitativo. Por un lado, está la verificación a cargo de los participantes, lo que implica que el investigador lleva resúmenes de los hallazgos a los participantes clave del estudio con el propósito de determinar si los hallazgos son reflexiones precisas de sus experiencias. Por otro lado, se tiene la triangulación de los datos obtenidos de varias fuentes o de varios individuos. Creswell (2007) afirma que este procedimiento es una práctica de análisis de datos común en donde el investigador construye evidencia para un código o un tema basado en varias fuentes o individuos. Por su parte, Sánchez Gómez (2015) sostiene que la triangulación debe ser considerada como el mayor nivel de integración, debido a que permite reconocer diferentes aspectos y aproximaciones de una misma realidad o fenómeno social, facilitando la convergencia. Complementariamente, Creswell (2007) menciona otra estrategia de validación que consiste en asegurarse si la información que presenta una perspectiva contraria a la evidencia establecida en realidad confirma la exactitud del análisis de los datos. Finalmente, Creswell (2007) sostiene que se puede optar por la estrategia que consiste en solicitar a pares externos que examinen los datos; estos pares deben estar familiarizados con investigación cualitativa al igual que con el área de la investigación.

\section{Conclusiones}

La discusión y análisis presentados con respecto a los conceptos específicos de los distintos componentes de la Metodología de la Investigación han pretendido brindar una visión global de esta práctica científica. Partiendo de la misma, se espera que la investigación sea entendida como una actividad de desarrollo profesional, que es capaz de traer beneficios a todas las partes involucradas. Además, a pesar de su inherente complejidad, la investigación no debe ser considerada como una tarea tan solo para grupos de élite. Esta actividad debe partir de un genuino deseo de hallar respuestas a interrogantes que el profesional se plantea en su labor diaria, lo que conduce a una planificación del proceso en la que se reconozcan las fortalezas y debilidades del investigador, con el fin de identificar áreas en las que se, sin perjuicio del valor de la investigación, se pueda buscar asesoría; esto ha de llevar a desarrollar un diseño de investigación adecuado a las necesidades de cada contexto, cuya ejecución permite alcanzar los objetivos planteados.

En lo que respecta a los enfoques mismos de la investigación, si bien es cierto la metodología cuantitativa ha sido tradicionalmente dominante - por la objetividad de los resultados y la relativa facilidad de réplica, la metodología cualitativa ha ido ganando espacio, especialmente por el progreso de las Ciencias Sociales, en las últimas décadas. Es importante enfatizar que, quizá como respuesta a una aparente disputa entre estos dos métodos, la investigación de métodos mixtos se ha presentado como una alternativa válida para los investigados que abandonan posturas metodologías y hasta filosóficas estrictas en miras de 
combinar las fortalezas de ambos métodos para desarrollar un diseño de investigación mixto que sirve de mejor manera a las necesidades y especificidades de cada estudio.

Es importante finalizar esta breve exposición con un exhorto a los investigadores principiantes, en todas áreas del conocimiento, a dejar de un lado posturas elitistas de la investigación, y plantearse el reto de dar respuesta a las preguntas que seguramente tienen mediante un proceso académicamente válido. Es innegable que la investigación es compleja; sin embargo, con dedicación, paciencia y esfuerzo, se logrará alcanzar resultados que traerán beneficios individuales y colectivos, siendo estos últimos los que deben prioritariamente motivar los procesos investigativos en todas las áreas del conocimiento.

\section{Bibliografía}

Bernal, C. (2013). Metodología de la Investigación (4ta ed.). Bogotá: Pearson Educación de Colombia.

Bloor, M. (2016). Addressing social problems through qualitative research. In D. Silverman (Ed.), Qualitative Research (pp 15 - 30). London: Sage Publications.

Brown, J. (1988). Understanding Research in Second Language Learning. Cambridge:

Cambridge University Press.

Brown, J. (2001). Using Surveys in Language Programs. Cambridge: Cambridge University Press.

Brown, J. (2014). Mixed Methods Research for TESOL. Edinburgh: Edinburgh University Press.

Creswell, J. (2007). Qualitative inquiry and research design: Choosing among five Approaches ( $2^{\text {nd }}$ ed.). Thousand Oaks, CA: Sage.

Creswell, J. (2009). Research Design: Qualitative, Quantitative, and Mixed Methods Approaches ( $3^{\text {rd }}$ ed.). Thousand Oaks, CA: Sage.

Creswell, J. \& Plano Clark, V. (2011). Designing and Conducting Mixed Method Research (2 ${ }^{\text {nd }}$ ed.). Thousand Oaks, CA: Sage.

Davies, K. (1995). Qualitative theory and methods in applied linguistics research. TESOL Quarterly 2 (29): 427- 453.

Dornyei, Z. (2007). Research Methods in Applied Linguistics: Quantitative, Qualitative, and Mixed Methodologies. Oxford: Oxford University Press.

Etikan, I., Abubakar, S., \& Sunusi, R. (2016). Comparison of Convenience Sampling and Purposive Sampling. American Journal of Theoretical and Applied Statistics, 5(1), 1-4. doi: 10.11648/j.ajtas.20160501.11

Erzberger, C. \& Kelle, U. (2003). Making inferences in mixed methods: the rules of Integration. In A. Tashakkori \& C. Teddie (Eds.), Handbook of Mixed Methods in Social \& Behavioral Research (pp. 457 - 488). Thousand Oaks, CA.: Sage.

Flick, V. (2014). La gestión de la calidad en la Investigación Cualitativa. Madrid: Morata.

Garrat, D. \& Li, Y. (2005). The foundations of experimental/empirical research methods. In B. Somekh \& C. Lewin (Eds.), Research Methods in the Social Sciences (pp 198 - 206). London: Sage.

Hatch, E. \& Lazaraton, A. (1991). The Research Manual: Design and Statistics for Applied Linguistics. New York: Newbury House. 
Gheitasi, P. \& Lindgren, E. (2015). Broadening the understanding of the language classroom: Mixed Methods. In Lindgren, E. \& Enever, J. (Eds.), Språkdidaktik: Researching Language Teaching and Learning (pp. 21 - 30). Umeå: Umeå University.

Johnson, R. \& Christensen, L. (2004). Education Research: Quantitative, Qualitative, and Mixed Approaches ( $2^{\text {nd }}$ ed.) Boston, MA: Allyn and Bacon.

Lazaraton, A. (2005). Quantitative Research Methods. In E. Hinkel (Ed.), Handbook of Research in Second Language Teaching and Learning (pp. 209 - 224). Mawah, NJ: Laurence Erlbau.

Lincoln, Y. \& Guba, E. (1985). Naturalistic inquiry. Beverly Hills, CA: Sage.

Mackey, A. \& Gass, S. (2005). Second Language Research: Methodology and Design. Mahwah, NJ: Lawrence Erlbaum Associates, Inc.

McKay, S. (2006). Researching Second Language Classrooms. Mahwah, NJ: Laurence Erlbaum.

Mertens, D. (2005). Research and Evaluation in Education and Psychology: Integrating Diversity with Quantitative, Qualitative, and Mixed Methods ( $2^{\text {nd }}$ ed.). Thousand Oaks, CA: Sage.

Miller, W. \& Crabtree, F. (1999). Depth interviewing. In B.F. Crabtree and W.L. Miller (Eds.), Doing Qualitative Research (pp. 89 - 107). London: Sage.

Miller, J. \& Glassner, B. (2016). The 'inside' and the 'outside': Finding realities in interviews. In D. Silverman (Ed.), Qualitative Research (pp 51 - 66). London: Sage Publications.

Onwuegbuzie, A. \& Leech, N. (2005). On becoming a pragmatic researcher: The Importance of combining quantitative and qualitative research methodologies. International Journal of Social Research Methodology 8(5): 575-587.

Sánchez Gómez, M. (2015). La dicotomía cualitativo-cuantitativa: posibilidades de integración y diseños mixtos. Campo Abierto, 11 - 30.

Sandelowsky, M. (2003). Tables or tableaux? The challenges of writing and reading mixed methods studies. In A. Tashakkori and C. Teddie (Eds.), Handbook of Mixed Methods in Social \& Behavioral Research (pp. 321 - 350). Thosand Oaks, CA. Sage.

Seidman, I. (1998). Interviewing as Qualitative Research: A guide for Researches in Education and the Social Sciences ( $2^{\text {nd }}$ ed.). New York: Teachers College Press.

Schwandt, T. (2000). Three epistemological stances for qualitative inquiry. In N.K.

Denzin and Y.S. Lincoln (Eds), Handbook of Qualitative Research (2 ${ }^{\text {nd }}$ ed.) (pp. 189 - 213). Thousand Oaks, CA: Sage. 